\title{
User-Centered Design of Mobile Wellness Applications
}

\author{
Aino Ahtinen \\ Tampere University of Technology \\ Korkeakoulunkatu 1, \\ 33720 Tampere, Finland \\ $+358504835602$ \\ aino.ahtinen@tut.fi
}

\begin{abstract}
The $\mathrm{PhD}$ research explores the design space of mobile wellness applications by utilizing design research methods. Several case studies are included in the research, e.g. [1],[2],[3],[4]. The main focus of the research is on the aspects, considerations and solutions related to motivational and persuasive mobile wellness applications. The research draws from the multifaceted combination of theories of human behavior, motivation and knowledge of technological possibilities, especially from the user experience perspective.
\end{abstract}

\section{Introduction}

The global concern of an increasing number of health problems due to the modern lifestyle is well-known - people around the world increasingly suffer from obesity etc. These cause a variety of problems at both personal and societal levels. Mobile technologies have been harnessed to support and motivate people in their wellness processes, and there is already plenty of research conducted in the domain. Most of the research and design on the domain has been conducted in the western, developed areas. One of our focuses is cross-cultural, combining research findings from two extremely different areas. The purpose is to study the similarities and differences as regards to wellness applications in these cultural contexts, and how should these affect to the design of them. Another theme of the research, which the domain is also lacking, is the long-term usage of wellness applications, i.e. what aspects characterize the use of different wellness technologies in a long time period? This paper describes the motivation, methodology and outcomes of these research cases.

\section{Cross-Cultural Factors on Wellness}

The design research process consists of several phases: explorative field studies, concept design iterations and concept evaluation. All phases were conducted in Finland and India. As data collection methods we used in-depth interviews, technology probe and participatory design tasks. Based on the findings from the field studies we built a concept model of a motivating mobile wellness application meant for the long-term use. The analysis of the results is still under progress. So far, one article concerning the design principles has been published [4]. Differences between the cultural contexts were found in the wellness definitions of the participants, their wellness activities and spaces, and the attitudes towards goal setting. Similarities were the wish for an interactive wellness application to keep up the curiosity towards the wellness activity, the application to provide interpretation instead of raw facts only, and the perceived high effort of manual input required by the used technology probe. The field study findings, as well as the concept model and its evaluation results in both contexts, are described in detail in the recently submitted journal article.

Copyright is held by the author/owner(s).

MobileHCI'09, September 15 - 18, 2009, Bonn, Germany.

ACM 978-1-60558-281-8.

\section{Long-Term Use of Wellness Applications}

Nuadu is a one-year health promotion trial with 120 participants using a set of available wellness technologies. In the trial we had a unique opportunity to study and compare the user experiences and motivational aspects of wellness technologies during a long time period with real consumers, who all are potential users of the wellness technologies. The technology toolkit consisted of seven wellness technologies, including wearable, mobile and Web-based solutions. The user experience data was collected with application logs, online questionnaires and telephone interviews in several phases of the trial. The findings of the use of mobile applications in the first phase of the trial have already been published [2]. In general, the applications got a positive response among the participants. In the beginning, many participants seemed to try out different functionalities due to curiosity and because they were looking for the most suitable solutions for themselves. In the end of the period, the usage habits and personal goals had shaped towards more practical and needs related usage, and active user groups had sprang up for each application. The analysis of the data during the whole trial is under progress, and will probably focus on how users with different profiles took the applications in to use, how their usage during a long time period changed, and what does the findings mean for the design of motivating wellness applications.

\section{Conclusions}

The research has quite wide focus, which on the other hand is a benefit, but brings also challenges for drawing the conclusions. The thesis worker would like to get feedback about that concern. She would also be happy to get feedback about the validity of the work done so far, and possible further case studies that would be required to complete the thesis.

\section{REFERENCES}

[1] Ahtinen, A., Isomursu, M. Huhtala, Y., Kaasinen, J., Salminen, J. \& Häkkilä, J. Tracking Outdoor Sports - User Experience Perspective. AmI 2008.

[2] Ahtinen, A., Mattila, E., Väätänen, A., Hynninen, L., Koskinen, E., Salminen, J. \& Laine, K. User Experiences of Mobile Wellness Applications in Health Promotion. Pervasive Health 2009.

[3] Ahtinen, A., Mäntyjärvi, J. \& Häkkilä, J. Using Heart Rate Monitors for Personal Wellness - The User Experience Perspective. EMBC 2008.

[4] Ahtinen, A., Ramiah, S., Blom, J. \& Isomursu, M. Design of Mobile Wellness Applications: Identifying Cross-Cultural Factors. OzChi 2008. 